\title{
Quasi-particle spectra of perovskites: Enhanced Coulomb correlations at surfaces
}

\author{
A. Liebsch \\ Institut für Festkörperforschung, Forschungszentrum Jülich, 52425 Jülich, Germany
}

(Dated: November 21, 2018)

\begin{abstract}
Photoemission spectra of the perovskites $\mathrm{Ca}_{x} \mathrm{Sr}_{1-x} \mathrm{VO}_{3}, \mathrm{Ca}_{x} \mathrm{La}_{1-x} \mathrm{VO}_{3}$, and $\mathrm{SrRuO}_{3}$ indicate that Coulomb correlations are more pronounced at the surface than in the bulk. To investigate this effect we use the dynamical mean field theory combined with the Quantum Monte Carlo technique and evaluate the multi-orbital self-energy. These systems exhibit different degrees of band filling and range from metallic to insulating. The key input in the calculations is the layer dependent local density of states which we obtain from a tight-binding approach for semi-infinite cubic systems. As a result of the planar character of the perovskite $t_{2 g}$ bands near the Fermi level, the reduced coordination number of surface atoms gives rise to a significant narrowing of the surface density of those subbands which hybridize preferentially in planes normal to the surface. Although the total band width coincides with the one in the bulk, the effective band narrowing at the surface leads to stronger correlation features in the quasi-particle spectra. In particular, the weight of the quasi-particle peak near $E_{F}$ is reduced and the amplitude of the lower and upper Hubbard bands is enhanced, in agreement with experiments.
\end{abstract}

\section{INTRODUCTION}

Strongly correlated materials are presently an active area of experimental and theoretical investigation [1, 2]. Angle-resolved photoemission spectroscopy in principle provides a complete map of the energy and momentum dependent quasi-particle states and has therefore been used to characterize the electronic properties of many fascinating systems. As a consequence of the finite escape depth of the emitted electron, however, photoemission spectra inevitably contain information on the electronic structure in the bulk and near the surface. Both the single- and many-particle features may depend on the distance from the surface. For example, even in the absence of any surface reconstruction the reduced coordination of surface atoms causes a characteristic oscillatory variation of the local density of states as a function of the layer index, with an appreciable effective band narrowing right at the surface [3]. In strongly correlated materials it is precisely the width and shape of this local density of states which, for a given set of on-site Coulomb and exchange energies, determine the details of the quasiparticle spectrum. Accordingly, the relative weights of the quasi-particle peak near the Fermi level and of the Hubbard bands may vary with distance from the surface. Additional effects can arise due to reconstruction of the lattice at the surface, due to more pronounced rotations or distortions of some of the atomic groups within the unit cell, surface phonons, and due to enhanced electronelectron interaction caused by less efficient surface screening processes.

Surface effects in photoemission from transition metal oxides have been observed in several systems. Fujioka et al. 4] studied $\mathrm{SrRuO}_{3}$ and noticed characteristic spectral variations caused by the frequency dependent mean free path of the photoelectron. A similar trend was found by Maiti et al. [5] for the series $\mathrm{Ca}_{x} \mathrm{La}_{1-x} \mathrm{VO}_{3}$. The latter data suggested the highly interesting situation of a metallic bulk coexisting with an insulating surface layer. Re- cently, Maiti et al. [6] and Sekiyama et al. 7] performed photoemission measurements on $\mathrm{Ca}_{x} \mathrm{Sr}_{1-x} \mathrm{VO}_{3}$ using a wide range of photon energies. These data also reveal striking effects associated with the varying amounts of bulk and surface contributions to the spectra. Typically, the valence bands in these systems consist of a coherent peak near $E_{F}$ derived from the partially filled transition metal $t_{2 g}$ bands, and a satellite feature corresponding to the lower Hubbard band. Inverse photoemission spectra reveal an analogous Hubbard band above $E_{F}$. In the measurements cited above, the weight of the coherent peak diminishes for shorter escape depth, while the satellite features (the so-called incoherent peaks) become more intense. Photoemission spectra exhibiting a relatively larger surface contribution therefore are more strongly correlated than bulk spectra.

This explains the puzzling behavior seen in early photoemission work on $\mathrm{Ca}_{x} \mathrm{Sr}_{1-x} \mathrm{VO}_{3}$ 8, 9, 10] in which $\mathrm{Ca}$ doping caused a significant suppression of intensity near $E_{F}$ and therefore appeared to drive the system close to a Mott transition. These results were at odds with the metallic behavior found in various thermodynamic measurements independently of Ca concentration 11]. Separating bulk and surface contributions by using different photon energies, Maiti et al. 6] and Sekiyama et al. 7] demonstrated that the bulk emission from $\mathrm{SrVO}_{3}$ and $\mathrm{CaVO}_{3}$ is quite similar, in agreement with the lowfrequency bulk probes and recent theoretical work 12]. The surface spectra of both materials, however, are considerably more correlated.

A related example is $\mathrm{Sr}_{2} \mathrm{RuO}_{4}$ for which previous photoemission spectra seemed to contradict bulk de Haasvan Alphen measurements 13]. Recent experimental and theoretical work proved, however, that this discrepancy can be resolved by taking into account the lattice reconstruction at the surface of $\mathrm{Sr}_{2} \mathrm{RuO}_{4}$ which leads to significant changes in the photoemission spectra 14].

To derive reliable information on bulk properties of strongly correlated systems using photoemission it 
clearly is desirable to identify single- and many-electron effects associated with the surface. In the present work we study these effects for three perovskite materials with widely different band fillings: the metallic compounds $\mathrm{Ca}_{x} \mathrm{Sr}_{1-x} \mathrm{VO}_{3}\left(d^{1}\right)$ and $\mathrm{SrRuO}_{3}\left(d^{4}\right)$, and the series $\mathrm{Ca}_{x} \mathrm{La}_{1-x} \mathrm{VO}_{3}$ which is insulating for $x=0\left(d^{2}\right)$, but metallic for $x=0.5\left(d^{1.5}\right)$. We evaluate the quasi-particle self-energy using the dynamical mean field theory based on the multi-orbital Monte Carlo method 2, 15, 16]. The important input in these many-body calculations is the layer dependent local density of states which we derive from a tight-binding scheme for semi-infinite systems [3]. We show that the surface leads to an effective narrowing of the density of states of those bands hybridizing mainly in atomic planes normal to the surface. As a result, correlation effects at the surface are more pronounced than in the bulk. Such a trend had first been predicted by Potthoff and Nolting [17 who studied the metal-insulator phase diagram for a semi-infinite simple cubic $s$ band at half filling. Here we calculate the self-energy for multiband systems using realistic local densities of states for several cubic perovskite materials and find qualitative agreement with photoemission data [4, 5, 6, 7]. Preliminary results on $\mathrm{SrVO}_{3}$ were published earlier [18].

This paper is organized as follows. In Section II we focus on the single-particle electronic properties of $\mathrm{SrVO}_{3}$ which is can be taken as representative of perovskite materials. In particular, we discuss the evaluation of the layer dependent local density of states for semi-infinite $\mathrm{SrVO}_{3}$. Section III provides the main elements of the calculation of the multi-orbital self-energy in the bulk and at the surface. The quasi-particle spectra of $\mathrm{Ca}_{x} \mathrm{Sr}_{1-x} \mathrm{VO}_{3}$, $\mathrm{SrRuO}_{3}$, and $\mathrm{Ca}_{x} \mathrm{La}_{1-x} \mathrm{VO}_{3}$ are presented in Section IV. Section V contains the summary.

\section{ELECTRONIC STRUCTURE: SRVO $_{3}$}

In this section we discuss the bulk and surface electronic properties of $\mathrm{SrVO}_{3}$. This system can be considered as a prototype of a cubic perovskite material since its one-electron structure is relatively simple, with one $d$ electron per transition metal ion. The other systems can be qualitatively understood in terms of these properties by accounting for different occupations: $d^{4}$ for $\mathrm{SrRuO}_{3}$, and $d^{2-x}$ for $\mathrm{Ca}_{x} \mathrm{La}_{1-x} \mathrm{VO}_{3}$.

Self-consistent electronic structure calculations for bulk $\mathrm{SrVO}_{3}$ within the local density approximation (LDA) 19] show that the conduction bands near the Fermi level consist of three degenerate $t_{2 g}$ bands derived from $\mathrm{V}^{4+}\left(3 d^{1}\right)$ ions. The filled $\mathrm{O} 2 \mathrm{p}$ bands are separated from the $t_{2 g}$ levels by a gap of about $1 \mathrm{eV}$, and the cubic crystal field of the V-O octahedron shifts the $\mathrm{V} e_{g}$ bands above the $t_{2 g}$ bands. Because of the cubic symmetry, the $t_{2 g}$ bands can be represented via a tight-binding
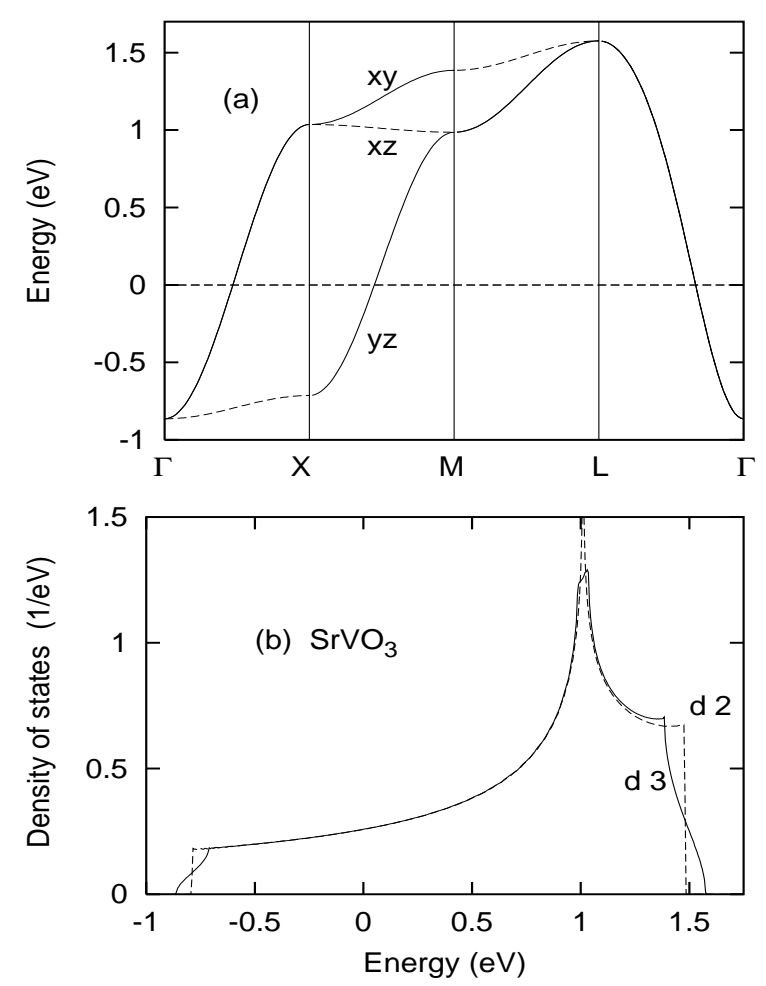

FIG. 1: (a) Tight-binding fit to LDA $t_{2 g}$ bulk bands of $\mathrm{SrVO}_{3}$ $\left(3 d^{1}\right)$. Solid lines: dispersion within the orbital plane; dashed lines: dispersion perpendicular to the orbital plane $\left(E_{F}=0\right)$. (b) Solid curve: density of states of $\mathrm{SrVO}_{3} t_{2 g}$ bulk bands. Dashed curve: analogous two-dimensional density of states obtained by neglecting inter-planar hopping integrals.

Hamiltonian with diagonal elements

$$
\begin{aligned}
h_{x y, x y}(k)= & e_{d}+t_{0}\left(c_{x}+c_{y}\right)+t_{1} c_{x} c_{y} \\
& +\left[t_{2}+t_{3}\left(c_{x}+c_{y}\right)+t_{4} c_{x} c_{y}\right] c_{z},
\end{aligned}
$$

where $c_{i}=2 \cos \left(k_{i} a\right), i=x, y, z$ and $a$ is the lattice constant. Cyclic permutations yield $h_{x z, x z}(k)$ and $h_{y z, y z}(k)$. The $t_{i}$ denote effective hopping integrals representing the V-O-V hybridization, where $t_{0,2}, t_{1,3}$, and $t_{4}$ specify the interaction between first, second and third neighbors, respectively. For symmetry reasons off-diagonal elements arise only between second and third nearest neighbors and are of the form $h_{x y, x z}(k)=-t_{1}^{\prime} s_{y} s_{z}-t_{2}^{\prime} c_{x} s_{y} s_{z}$, where $s_{i}=2 \sin \left(k_{i} a\right)$, i.e., they vanish at the highsymmetry points. Since the coefficients $t_{1,2}^{\prime}$ are very small we neglect these off-diagonal elements so that the energy bands are given by $\epsilon_{i}(k)=h_{i, i}(k)$, with $i=x y, x z, y z$. The tight-binding parameters $e_{d}$ and $t_{0} \cdots t_{4}$ can easily be found by fitting the LDA energies at high-symmetry points of the bulk Brillouin Zone.

Fig. 1 (a) shows the $t_{2 g}$ bulk bands of $\mathrm{SrVO}_{3}$ along the main symmetry directions. The conduction bands in the cubic perovskite structure consist of three nearly non-hybridizing $t_{2 g}$ bands. Each of these bands is approximately two-dimensional representing weakly cou- 
pled atomic planes. Typically, the inter-planar band width is about 20 times smaller than the intra-planar band width. To indicate the pronounced planar character of these bands we denote via solid lines the strong dispersion within the plane spanned by an orbital and by dashed lines the much weaker out-of-plane dispersion. According to this nearly two-dimensional electronic structure, the bulk density of states $\rho_{b}(\omega)$ exhibits the characteristic main peak related to the van Hove singularity at the $\mathrm{X}$ point of the Brillouin Zone. This is shown in Fig. 1 (b) where the bulk density of states is compared with the two-dimensional density obtained by setting the inter-planar hopping integrals $t_{2,3,4}$ equal to zero. The asymmetric shape of both distributions follows from the second-neighbor hopping terms $\sim t_{1}$. The overall shape of the bulk density agrees well with the one obtained from LAPW calculations [19].

Note that as a result of the $3 d^{1}$ configuration, the Fermi surface of $\mathrm{SrVO}_{3}$ consists of three nearly perfect intersecting cylinders containing the $d_{x y}, d_{x z}$ and $d_{y z}$ states. This peculiar shape was recently observed also in de Haas-van Alphen measurements of $\mathrm{CaVO}_{3}$ [20] in spite of pronounced orthorhombic distortions.

In the cubic environment, the three $t_{2 g}$ bands have identical density of states. At the surface, the bulk degeneracy is lifted since only the $d_{x y}$ band exhibits strong dispersion within the plane of the surface (the $z$ direction specifies the surface normal) whereas the $d_{x z}$ and $d_{y z}$ bands disperse primarily within atomic planes perpendicular to the surface plane. Thus, the local density of states of the $d_{x y}$ band in the first layer is similar to the bulk density, while that of the $d_{x z, y z}$ bands is modified by the reduced coordination number in the $z$ direction. To evaluate these surface densities we use a Green's function formalism [3] for semi-infinite tight-binding systems.

Neglecting again the weak hybridization between $t_{2 g}$ states, the local density of states of band $i \equiv x y, x z, y z$ is given by

$$
\rho_{i, n}(\omega)=\frac{1}{\pi} \sum_{k_{\|}} \operatorname{Im} G_{i, n}\left(k_{\|}, \omega\right)
$$

where $n \geq 1$ denotes the layer index and the Green's function can be conveniently determined from the expression

$$
G_{i, n}\left(k_{\|}, \omega\right)=\frac{i}{\mu}\left[1+\left(\frac{i \mu+\Omega}{2 T_{i}}\right)^{m}\left(\frac{i \mu+\Omega-\Delta}{i \mu-\Omega+\Delta}\right)\right]
$$

where $m=2 n-2, \mu=\left(4 T_{i}^{2}-\Omega\right)^{1 / 2}$ and $\Omega=\omega-W_{i}$. The parameter $\Delta$ denotes a surface potential. $W_{i}$ and $T_{i}$ represent intra- and inter-planar contributions to the $t_{2 g}$ band energies:

$$
\begin{aligned}
& W_{x y}=e_{d}+t_{0}\left(c_{x}+c_{y}\right)+t_{1} c_{x} c_{y} \\
& W_{x z}=e_{d}+t_{0} c_{x}+t_{2} c_{y}+t_{3} c_{x} c_{y} \\
& W_{y z}=e_{d}+t_{0} c_{y}+t_{2} c_{x}+t_{3} c_{x} c_{y}
\end{aligned}
$$

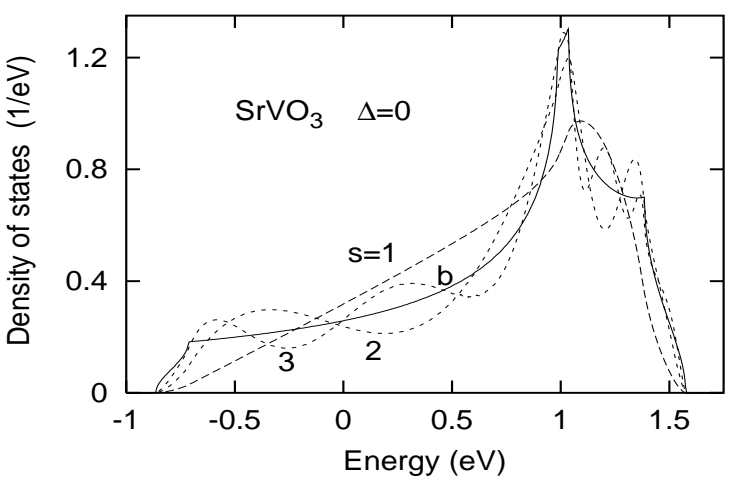

FIG. 2: Layer dependent local density of states $\rho_{i, n}(\omega)$ of outof-plane $d_{x z, y z}$ bands for first three atomic planes of $\mathrm{SrVO}_{3}$ $\left(E_{F}=0\right)$. The surface potential $\Delta$ is set equal to zero. Solid curve: isotropic bulk density of states $\rho_{b}(\omega)$. The local density of the intra-planar $d_{x y}$ states (not shown) is similar to the bulk density, even in the surface layer.

and

$$
\begin{aligned}
& T_{x y}=t_{2}+t_{3}\left(c_{x}+c_{y}\right)+t_{4} c_{x} c_{y} \\
& T_{x z}=t_{0}+t_{1} c_{x}+t_{3} c_{y}+t_{4} c_{x} c_{y} \\
& T_{y z}=t_{0}+t_{1} c_{y}+t_{3} c_{x}+t_{4} c_{x} c_{y} .
\end{aligned}
$$

How $\mathrm{SrVO}_{3}$ is terminated at the surface, especially the charge state of the $\mathrm{V}$ ions in the first layer, is not yet known. Thus, we assume all tight-binding parameters to coincide with those in the bulk. The surface potential is chosen to ensure charge neutrality (see below).

Fig. 2 compares the layer dependent local density of states $\rho_{i, n}(\omega)$ of the $d_{x z, y z}$ bands with the isotropic bulk density $\rho_{b}(\omega)$. The $d_{x z, y z}$ density in the first plane is seen to be less sharply peaked and more narrow than $\rho_{b}(\omega)$ although their total widths are identical. In particular, $\rho_{x z, 1}(\omega)$ rises almost linearly below $E_{F}$ in contrast to the plateau-like shape of $\rho_{b}(\omega)$. Thus, the surface spectral weight is reduced at low and high frequencies and enhanced at intermediate frequencies. The local density of $d_{x z, y z}$ states in the deeper lying layers approaches the bulk density rather quickly, the main effect consisting in an oscillatory distortion of the spectral shape rather than any appreciable band narrowing. The rapid convergence towards the bulk density is to be expected because of the tight-binding character of the $t_{2 g}$ bands resulting from the short range of the hopping integrals. The local density of the in-plane $d_{x y}$ states depends only weakly on the layer index and nearly coincides with the bulk density even in the first layer (see below).

It would of course be desirable to perform selfconsistent electronic structure calculations for semiinfinite $\mathrm{SrVO}_{3}$ since they should provide a more accurate description of the density of states in the surface region. Nevertheless, we believe that the key effect discussed here within the simplified tight-binding approach, namely, the preferential band narrowing of the $d_{x z, y z}$ states, will hold 
quite generally.

The single particle properties of the remaining perovskite systems discussed in this work are closely related to those of $\mathrm{SrVO}_{3}$. They differ essentially by the degree of filling of the $t_{2 g}$ bands, and by the deviation from cubic symmetry via orthorhombic distortions of the oxygen octahedra surrounding the transition metal ions. These distortions primarily broaden the van Hove singularity of the density of states but have only a minor effect on the overall width of the $t_{2 g}$ bands [12, 21]. We discuss these differences in more detail in Section IV.

\section{QUASI-PARTICLE SPECTRA}

To analyze the experimental photoemission data we evaluate the quasi-particle spectra by taking into account local Coulomb interactions. According to the semiinfinite one-electron properties discussed above we are dealing with a non-isotropic system where two narrow $d_{x z, y z}$ bands interact with a wider $d_{x y}$ band. In the first atomic layer this difference in effective band width is most pronounced and it diminishes rapidly towards the interior of the system. This situation is reminiscent of the one in the layer perovskite $\mathrm{Sr}_{2} \mathrm{RuO}_{4}$, which essentially consists of $\mathrm{Ru}$ sheets containing two nearly onedimensional $d_{x z, y z}$ bands interacting with a wide intraplanar $d_{x y}$ band. The peculiar interesting feature of the latter system is the fact the on-site Coulomb energy lies between the single-particle widths of the non-degenerate $t_{2 g}$ bands: $W_{x z, y z}<U<W_{x y}$ [22]. In the present case, on the other hand, the difference between the $d_{x z, y z}$ and $d_{x y}$ bands at the surface is less pronounced so that $W_{i}<U$ for all three bands. Nevertheless, since in the first layer the effective width of $d_{x z, y z}$ states is reduced, the effect of Coulomb correlations on the surface bands should be stronger than on the wider bulk bands.

The key quantity characterizing the effect of Coulomb correlations on quasi-particle spectra is the self-energy which we evaluate here using the dynamical mean field theory (DMFT) [2, 15, 16]. A full description of correlations near the surface would be exceedingly complicated since, in principle, it would require a mixed momentum/real space approach in order to handle the loss of translational symmetry normal to the surface. This could be accomplished using a cluster formalism in which the semi-infinite system is represented via a slab of finite thickness. Unfortunately, the planar character of the $t_{2 g}$ states leads to a slowly convergent local density of $d_{x z, y z}$ states, with many spurious $1 / \sqrt{\omega}$ van Hove singularities stemming from the quasi-one-dimensional hopping along atomic rows parallel to the surface. On the other hand, a cluster generalization of the DMFT is feasible today only for very small cluster size.

To achieve a qualitative understanding of correlations at $\mathrm{SrVO}_{3}$ surfaces we ignore the momentum variation of the self-energy and assume that, for a given layer, it depends only on the local density of states within that layer
[17. Since we neglect the weak hybridization between $t_{2 g}$ orbitals, the self-energy is diagonal in orbital space. To evaluate the self-energy elements $\Sigma_{i}(\omega)$ we use the dynamical mean field approach, in which $\Sigma_{i}$ is a functional of the bath Green's function $\mathcal{G}_{i}^{-1}=G_{i}^{-1}+\Sigma_{i}$, where the local $G_{i}$ is given by

$$
G_{i}\left(i \omega_{n}\right)=\int_{-\infty}^{\infty} d \omega \frac{\rho_{i}(\omega)}{i \omega_{n}+\mu-\Sigma_{i}\left(i \omega_{n}\right)-\omega} .
$$

The Matsubara frequencies are denoted by $\omega_{n}$ and $\mu$ is the chemical potential. On-site Coulomb correlations are treated using the self-consistent multiband Quantum Monte Carlo (QMC) method (for a review, see Ref. [2]). The temperature of the simulation was $125 \mathrm{meV}$ $(\beta=8)$. Several runs using 64 imaginary time slices and $10^{5}$ Monte Carlo sweeps were carried out. The quasiparticle density of states $N_{i}(\omega)=-\operatorname{Im} G_{i}(\omega) / \pi$ was obtained via maximum entropy reconstruction [23].

In principle, we are now faced with a set of coupled impurity problems, where the layer-dependent baths determine the self-energies of each atomic plane. This problem could be solved iteratively until self-consistency is achieved and a common chemical potential is found for the bulk and at the surface. Unfortunately, for multiband systems this iteration procedure would be computationally extremely demanding and will be neglected here. On the other hand, previous work [17] showed that the self-energy is mainly governed by local Coulomb correlations and that further changes due to interlayer coupling are rather small. Our assumption of charge neutrality within the surface layer, and the fact that correlations in the bulk and at the surface do not differ substantially, most likely also reduce the importance of interlayer effects. The key layer dependent input in our QMC-DMFT calculation is therefore the one-electron local density of states. For a given set of local Coulomb and exchange energies the width and shape of this local density then determines the details of the quasi-particle spectrum.

\section{RESULTS AND DISCUSSION}

\section{A. $\mathrm{SrVO}_{3}$}

The layer dependent local density of states of $\mathrm{SrVO}_{3}$ shown in Fig. 1(b) is calculated in the absence of any surface potential. Thus, the occupation numbers also vary with layer index. However, since local Coulomb correlations are very sensitive to the degree of band filling, it seems appropriate to enforce charge neutrality by adjusting $\Delta$ accordingly. Fig. 3 shows the resulting local density of states for the surface layer. Although the main peak now coincides more with the bulk van Hove singularity, the effective narrowing is similar to the one in Fig. 1(b). Note that the surface potential is sufficiently weak so that no surface states are split off below the band. Thus, the total band width is the same as in the bulk. No surface 


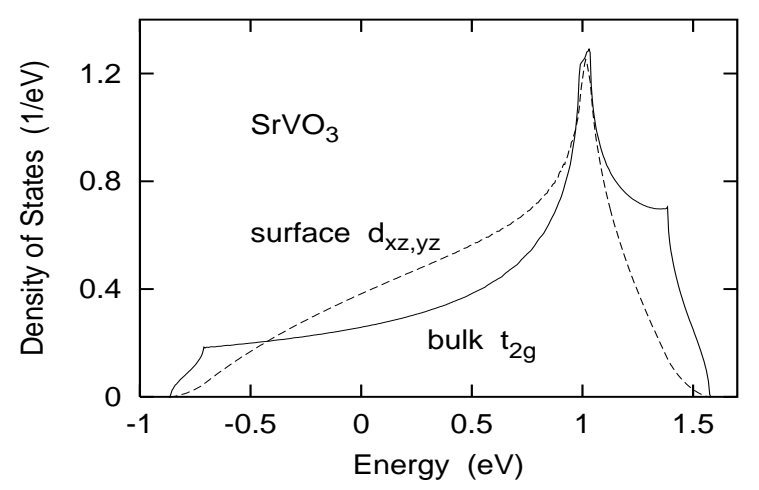

FIG. 3: Local density of $d_{x z, y z}$ states of first layer of $\mathrm{SrVO}_{3}$ $\left(d^{1}\right)$ in the presence of a weak surface potential $\Delta$ to ensure charge neutrality (dashed curve). The $t_{2 g}$ bulk density (solid curve) is shown for comparison. The density of the in-plane $d_{x y}$ states in the first layer (dotted curve) is nearly identical to the bulk density.

potential is needed for the $d_{x y}$ states since their local density is practically identical to the bulk $t_{2 g}$ density.

Fig. 4 (a) shows the bulk quasi-particle density of states of $\mathrm{SrVO}_{3}$ for two Coulomb energies in the region where the Hubbard bands which are seen as satellites in photoemission begin to emerge: $U=4.0 \mathrm{eV}$ and $4.3 \mathrm{eV}$. The exchange energy is $J=0.7 \mathrm{eV}$ [25]. These results show that in the bulk $U$ must be larger than $4 \mathrm{eV}$ to obtain the satellite observed in photoemission spectra [6, 7]. The peak near $2 \mathrm{eV}$ above $E_{F}$ agrees with inverse photoemission data 10 . Although for $U=4 \mathrm{eV}$ there is considerable correlation-induced band narrowing and an emerging satellite shoulder, the larger $U$ to yields an even narrower coherent feature near $E_{F}$, with the missing weight shifted to the lower and upper Hubbard bands.

Note that $N_{b}\left(E_{F}\right)=\rho_{b}\left(E_{F}\right)$ which follows (at $\left.T=0\right)$ from the local approximation implicit in the DMFT for isotropic systems [26]. The most recent photoemission data 7] confirm this result. The bulk spectra shown in Fig. 4 (a) qualitatively agree with DMFT-LDA results for $\mathrm{SrVO}_{3}$ by Nekrasov et al. 12] who employed somewhat larger values for $U$ and $J$. They are also consistent with previous spectra for the $3 d^{1}$ perovskite $\mathrm{La}_{x} \mathrm{Sr}_{1-x} \mathrm{TiO}_{3}$ 27. which exhibits a similar $t_{2 g}$ bulk density of states.

The surface quasi-particle spectra for $\mathrm{SrVO}_{3}$ are shown in Fig. 4(b) and (c). The lower Hubbard peak of the $d_{x z, y z}$ states in (c) is clearly visible already for $U=4 \mathrm{eV}$ because of the narrower local density of states in the first layer. A larger $U$ shifts the satellite to higher binding energies. The comparison with the spectra shown in Fig. 4 (a) demonstrates that correlation effects for a fixed value of $U$ are stronger at the surface than in the bulk: The coherent peak near $E_{F}$ is narrower at the surface and the incoherent satellite feature is more pronounced than in the bulk, in agreement with experiment [4, 6, 7].

The surface quasi-particle density of $d_{x y}$ states in Fig. 4 (b) is intermediate between $N_{b}(\omega)$ and $N_{s}(\omega)$ for
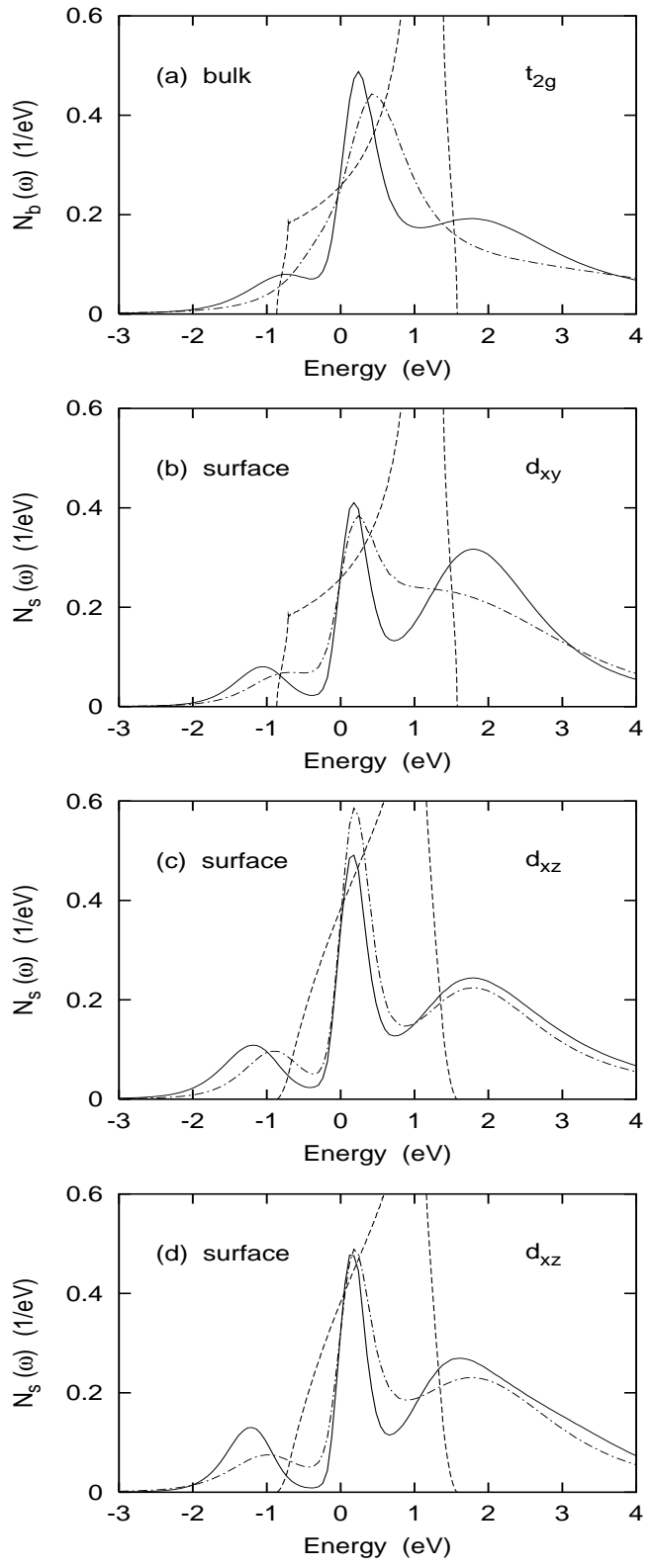

FIG. 4: Quasi-particle density of states $N_{i}(\omega)$ of $\mathrm{SrVO}_{3}$ $\left(d^{1}\right)$ derived from DMFT. (a) bulk $t_{2 g}$ states; (b) surface $d_{x y}$ states; (c) surface $d_{x z, y z}$ states; (d) fictitious isotropic surface $d_{x z, y z}$ states (see text). Solid curves: $U=4.3 \mathrm{eV}$, dot-dashed curves: $U=4.0 \mathrm{eV}(J=0.7 \mathrm{eV})$. Dashed curve: bare densities of states (see Fig. 3).

$d_{x z, y z}$. Although there is little single-electron hybridization between $t_{2 g}$ bands, the local Coulomb interaction mixes them so that the $d_{x y}$ surface spectrum involves contributions arising from the more strongly correlated $d_{x z, y z}$ states. Another consequence of the anisotropic surface self-energy is the correlation-induced charge transfer between subbands 222. Here, we find that 0.06 electrons are shifted from the $d_{x y}$ to the $d_{x z, y z}$ bands. Also, the quasi-particle partial densities of states at $E_{F}$ do not 
need to coincide with the bare partial densities. The coupling between narrow and wide bands is a genuine multiband effect and underlines the fact that single-particle bands in the presence of local Coulomb interactions cannot be considered independently.

For illustrative purposes we show in Fig. 4 (d) the analogous quasi-particle spectra for a hypothetical isotropic system whose density of states is given by the surface $\rho_{x z, y z}$. Evidently, correlation effects are slightly more pronounced than at the surface of $\mathrm{SrVO}_{3}$ where two such narrow bands interact with the wider $d_{x y}$ band. All spectra shown Fig. 4 are based on single-particle densities of identical total width but with progressively more orbital components exhibiting narrower spectral shape. Accordingly, the weight of the quasi-particle peak near $E_{F}$ decreases systematically and the upper and lower Hubbard bands become successively more intense.

Note that the quasi-particle spectra in Fig. 4 are highly asymmetric and that the gap between the central peak and the lower satellite appears at smaller $U$ than the upper gap. This behavior is a consequence of the asymmetric bulk and surface density of states, and of the low band filling in this system ( $1 / 6$ per spin band). Thus, despite the typical overall 3-peak structure which is characteristic of many highly correlated quasi-particle distributions, their is a large degree of spectral detail which is characteristic of this particular material.

We also point out that the many-body reduction of the quasi-particle band width is very much larger than the surface-induced one-electron band narrowing. On the other hand, since the on-site Coulomb energy is not far from the critical value for a metal-insulator transition, the band narrowing substantially enhances the influence of correlations at the surface.

It would be interesting to perform angle-resolved photoemission measurements to determine the correlationinduced band narrowing of the $\mathrm{SrVO}_{3} t_{2 g}$ bands. For instance the energy at $\bar{\Gamma}$ should be only a few tenths of an $\mathrm{eV}$ below $E_{F}$ instead of $1 \mathrm{eV}$ as predicted by the LDA. Accordingly, the true $t_{2 g}$ bands should be considerably flatter than the single-particle bands. Also, measurements using polarized light could help to separate correlation effects in the $d_{x y}$ and $d_{x z, y z}$ bands.

\section{B. $\mathrm{CaVO}_{3}$}

In striking contrast to thermodynamic measurements earlier photoemission data on $\mathrm{Ca}_{x} \mathrm{Sr}_{1-x} \mathrm{VO}_{3}$ [8, 9, 10] indicated considerably more pronounced correlations in $\mathrm{CaVO}_{3}$ than in $\mathrm{SrVO}_{3}$. These observations even led to speculations as to whether $\mathrm{CaVO}_{3}$ might be close to at Mott transition. However, recent photoemission measurements taken over a wide range of photon energies [6, 7] demonstrated that this discrepancy can be resolved by carefully separating bulk and surface contributions to the spectra by using widely different photon energies. According to these results, the bulk emission from $\mathrm{CaVO}_{3}$ is quite similar to that from $\mathrm{SrVO}_{3}$, the $\mathrm{CaVO}_{3}$ spectra being only slightly more correlated. Thus, the new data are consistent with low-frequency bulk measurements.

While $\mathrm{SrVO}_{3}$ is a cubic perovskite with a $\mathrm{V}-\mathrm{O}-\mathrm{V}$ bond angle of $180^{\circ}$, the oxygen octahedra in $\mathrm{CaVO}_{3}$ are distorted, so that the $\mathrm{V}-\mathrm{O}-\mathrm{V}$ bond angle is reduced to $162^{\circ}$, implying a weaker indirect hopping between $d$ orbitals and a reduction of the $d$ electron band width. As the most recent LDA calculations for $\mathrm{CaVO}_{3}$ show [12], this decrease in total band width amounts to only about $4 \%$ (from 2.6 to $2.5 \mathrm{eV}$ ). A more striking consequence of the distortion is the considerable broadening of the van Hove singularity and the skewing of its spectral weight to lower frequencies. Similar results had been obtained in LDA calculations for $\mathrm{SrRuO}_{3}$ and $\mathrm{CaRuO}_{3}$ 21], where orthorhombic distortions also cause a narrowing of the $t_{2 g}$ bands and a broadening of the van Hove singularity. As the LDA-DMFT bulk calculations for $\mathrm{CaVO}_{3}$ by Nekrasov et al. 12] show, these changes of the density of states lead to a small reduction of the quasi-particle peak near $E_{F}$ and to slightly more intense Hubbard bands compared to those in $\mathrm{SrVO}_{3}$, in agreement with the data.

Considering the enhancement of Coulomb correlations at the $\mathrm{SrVO}_{3}$ surface discussed in the previous section, and the fact that in the bulk $\mathrm{CaVO}_{3}$ is already somewhat more correlated than $\mathrm{SrVO}_{3}$, it is now plausible that correlation effects should be even stronger at the $\mathrm{CaVO}_{3}$ surface, as indeed observed. Since the local Coulomb energy is not far from the critical value for a metalinsulator transition, the tendency for enhanced surface correlations in $\mathrm{CaVO}_{3}$ should in fact be stronger than for $\mathrm{SrVO}_{3}$. This effect is entirely a consequence of the one-electron band narrowing of the $d_{x z, y z}$ subbands. Additional effects could be caused by surface reconstruction or by more pronounced tilting of oxygen octahedra at the $\mathrm{CaVO}_{3}$ surface which would lead to weaker in-plane hopping for the $d_{x y}$ bands. For a more detailed analysis it would be necessary to carry out electronic structure calculations for the $\mathrm{CaVO}_{3}$ surface in order to study orthorhombic distortions and possible reconstructions. In addition, theoretical estimates of a possible increase of $U$ at the surface due to reduced screening would be useful.

\section{C. $\mathrm{SrRuO}_{3}$}

The ruthenates with perovskite-based crystal structure have attracted considerable attention during the past years because of a variety of fascinating properties. $\mathrm{SrRuO}_{3}$ is the only known ferromagnetic metal among the $4 d$ oxides, with a Curie temperature of $160 \mathrm{~K}$. The one-electron density of states is similar to that of $\mathrm{SrVO}_{3}$ except for the $d^{4}$ occupancy of the Ru derived $t_{2 g}$ bands 21]. In a perfect cubic system the Fermi level would nearly coincide with the van Hove singularity and the flat bands in this energy region. However, the oxygen octahedra in $\mathrm{SrRuO}_{3}$ are tilted by about $8^{\circ}$ which leads to a substantial broadening of the van Hove singularity. 


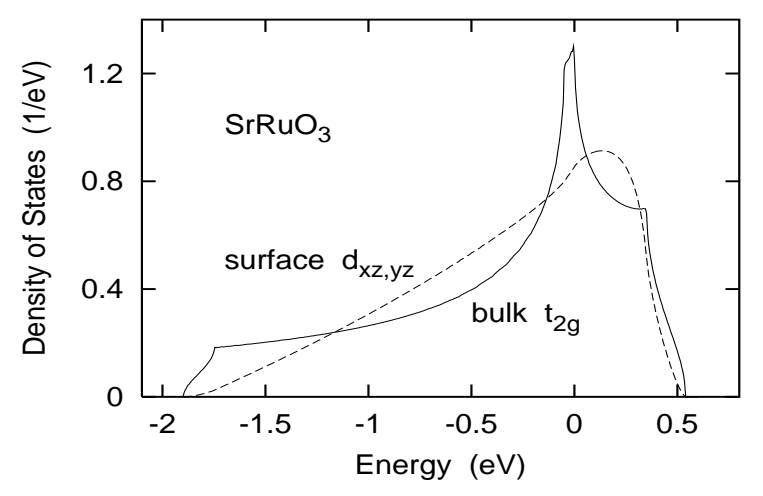

FIG. 5: Solid curve: isotropic bulk density of states of cubic $\mathrm{SrRuO}_{3}$; dashed curve: local density of $d_{x z, y z}$ states in first layer $\left(E_{F}=0\right)$.

Nevertheless, the density of states near $E_{F}$ remains large, giving rise to a large Stoner parameter. Doping with Ca causes further tilting of the oxygen octahedra. As a result the density of states at $E_{F}$ is reduced even further and the magnetism is easily suppressed. The layered ruthenate $\mathrm{Sr}_{2} \mathrm{RuO}_{4}$ is a paramagnetic metal and is currently intensively studied because of its unconventional p-wave superconductivity below $1.5 \mathrm{~K}[28$.

The unusual magnetic properties of the ruthenates have stimulated wide-ranging investigations of the correlation effects in these materials. Transport measurements [30] as well as theoretical studies [29] on $\mathrm{SrRuO}_{3}$ seemed to suggest non-Fermi-liquid behavior. Nevertheless, recent transport data on highly ordered samples 31] are consistent with Fermi-liquid theory, but demonstrate a remarkable sensitivity to thermal and impurity scattering.

Photoemission spectra on $\mathrm{SrRuO}_{3}[4]$ revealed marked spectral changes for different photon energies. At $1254 \mathrm{eV}$ emission from the $\mathrm{Ru} 4 d$ bands exhibits pronounced weight at $E_{F}$ and a maximum at about $1.5 \mathrm{eV}$ binding energy. At $100 \mathrm{eV}$, on the other hand, the spectral weight at $E_{F}$ is much smaller, and the intensity then grows down to $2 \mathrm{eV}$ binding energy. (The region farther below is dominated by $\mathrm{O} 2 p$ emission.) Thus, the relatively more important surface contribution caused by the shorter escape depth of the photoelectron makes the spectrum appear more correlated than at energies governed by bulk emission.

To investigate correlations in the bulk and at the surface of $\mathrm{SrRuO}_{3}$ we have applied the approach discussed above to the case of $d^{4}$ occupancy of the $t_{2 g}$ bands. For the case of cubic perovskite structure, i.e., neglecting the tilting of the $\mathrm{O}$ octahedra, the isotropic bulk density of states shown in Fig. 5 agrees qualitatively with results of LAPW calculations [21]. The local density of $d_{x z, y z}$ states in the first layer shows again the characteristic shift of weight from low and high frequencies to the intermediate range closer to $E_{F}$. The $d_{x y}$ surface density nearly
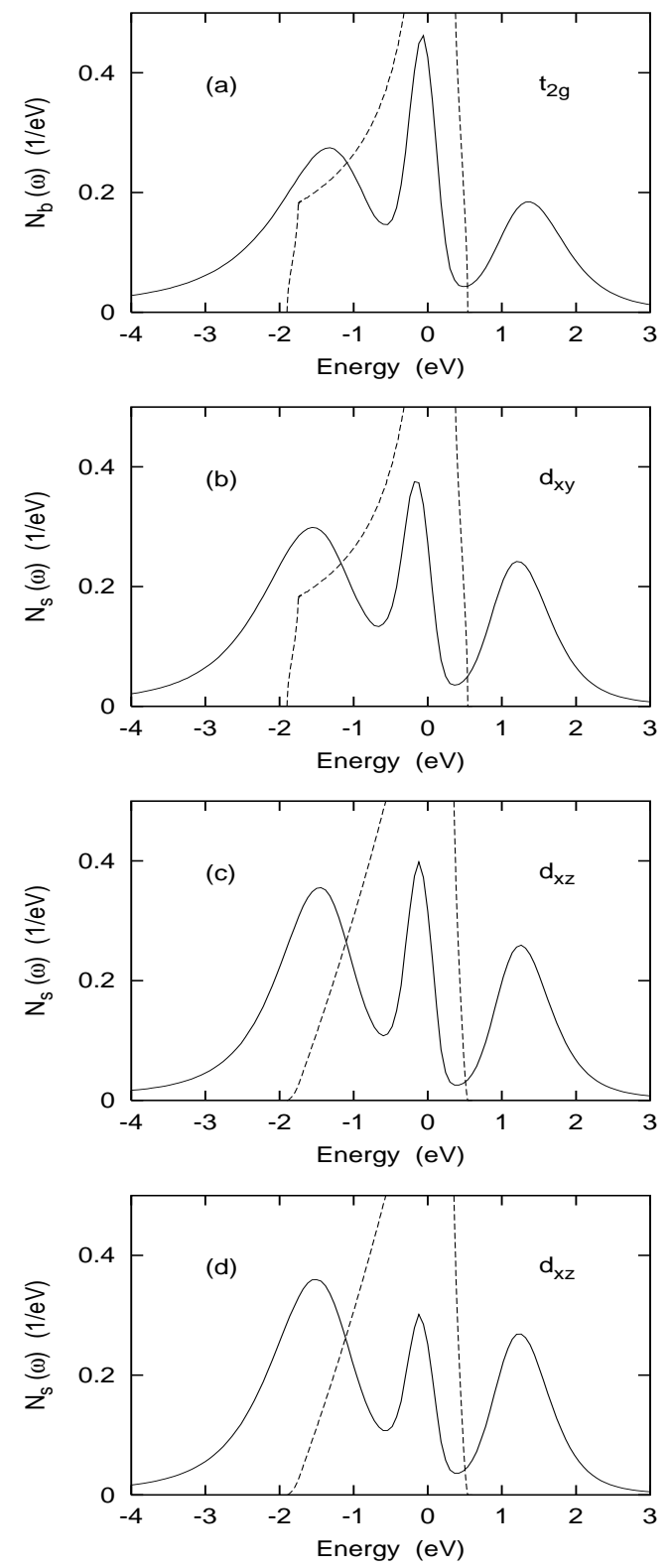

FIG. 6: Quasi-particle density of states $N_{i}(\omega)$ of $\mathrm{SrRuO}_{3}$ $\left(d^{4}\right)$ derived from DMFT. (a) bulk $t_{2 g}$ states; (b) surface $d_{x y}$ states; (c) surface $d_{x z, y z}$ states; (d) fictitious isotropic surface $d_{x z, y z}$ states (see text). Solid curves: $U=3.0 \mathrm{eV}, J=0.2 \mathrm{eV}$; dashed curves: bare densities of states (see Fig. 5).

coincides with the bulk $t_{2 g}$ density.

Fig. 6 shows the quasi-particle spectra obtained from DMFT-QMC calculations for $U=3.0 \mathrm{eV}$ and $J=$ $0.2 \mathrm{eV}$. As in the case of $\mathrm{SrVO}_{3}$, there is a clear progression of correlation effects as we go from the isotropic bulk $t_{2 g}$ spectra to the $d_{x y}$ and $d_{x z, y z}$ surface spectra. The hypothetical isotropic case based on the surface $d_{x z, y z}$ density (i.e., equating $\rho_{x y}$ with $\rho_{x z, y z}$ ), shown in Fig. 6 (d), is even more strongly correlated, with less spectral weight of the coherent feature near $E_{F}$ and accordingly larger 
upper and lower Hubbard bands. Again, all bare densities have the same total width. Thus, the differences between the bulk and surface quasi-particle spectra are entirely due to the different shapes of the densities. Note also that, in contrast to the results shown in Fig. 4 for $\mathrm{SrVO}_{3}, N_{b, s}\left(E_{F}\right)<\rho_{b, s}\left(E_{F}\right)$. This deviation is caused by the van Hove singularity at $E_{F}$ and our use of a rather high temperature (about $1450 \mathrm{~K}$ ) in the QMC calculations.

The results shown in Fig. 6 provide a qualitative explanation for the experimentally observed shift of spectral weight towards the lower Hubbard peak near $1.5 \mathrm{eV}$ binding energy upon lowering the photon energy from $1254 \mathrm{eV}$ to $100 \mathrm{eV}$ [4] and thereby enhancing the surface contribution to the photoemission spectra. Of course, as in the case of $\mathrm{SrVO}_{3}$, other surface effects, e.g., due to reconstruction, distortion of oxygen octahedra, etc., might also play a role and presumably would reduce not only the hopping between $d_{x z, y z}$ orbitals of neighboring layers but also between $d_{x y}$ states within the surface plane. These modifications of the electronic structure would lead to a further enhancement of surface correlation effects.

\section{D. $\mathbf{C a}_{x} \mathbf{L a}_{1-x} \mathrm{VO}_{3}$}

The perovskite series $\mathrm{Ca}_{x} \mathrm{La}_{1-x} \mathrm{VO}_{3}$ is very interesting in the context of surface versus bulk Coulomb correlations since $\mathrm{LaVO}_{3}(x=0)$ is a Mott insulator ( $d^{2}$ occupancy) [32] which readily becomes metallic upon increasing the Ca concentration to $x=0.5$ ( $d^{1.5}$ occupancy). Since doping with $\mathrm{Ca}$ leads to little structural changes and nearly constant Hubbard $U$ [33], the modifications of the electronic properties are almost entirely caused by the degree of band filling.

To investigate these effects, Maiti et al. [5] carried out photoemission measurements at $\mathrm{Ca}$ concentrations $x=0.0 \ldots 0.5$ and using XPS and VUV photon energies to distinguish bulk and surface contributions. They observed not only the bulk Mott transition at about $x=0.2$, but in addition a surface Mott transition at a slightly larger value of $x$. Thus, these data suggest the coexistence of a metallic bulk with an insulating surface layer. This finding is in conflict with predictions 17 for the simple-cubic half-filled $s$ band, but it is not clear at present to what extent the theoretical results are applicable to multiband systems at arbitrary occupancies. Moreover, the calculations in Ref. 17] were done for a linearized version of the DMFT in which low- and highfrequency excitations within the $s$ band are essentially decoupled.

We have calculated the quasi-particle spectra of $\mathrm{LaVO}_{3}$ and $\mathrm{Ca}_{0.5} \mathrm{La}_{0.5} \mathrm{VO}_{3}$ within multi-band QMC-DMFT. The bulk density of states is assumed to be similar to the one for cubic $\mathrm{SrVO}_{3}$, except for the filling of the $t_{2 g}$ bands. As shown in Fig. 7, the shape of the $d_{x z, y z}$ density in the surface layer differs for these two systems since we employ slightly different surface potentials in the semi-infinite
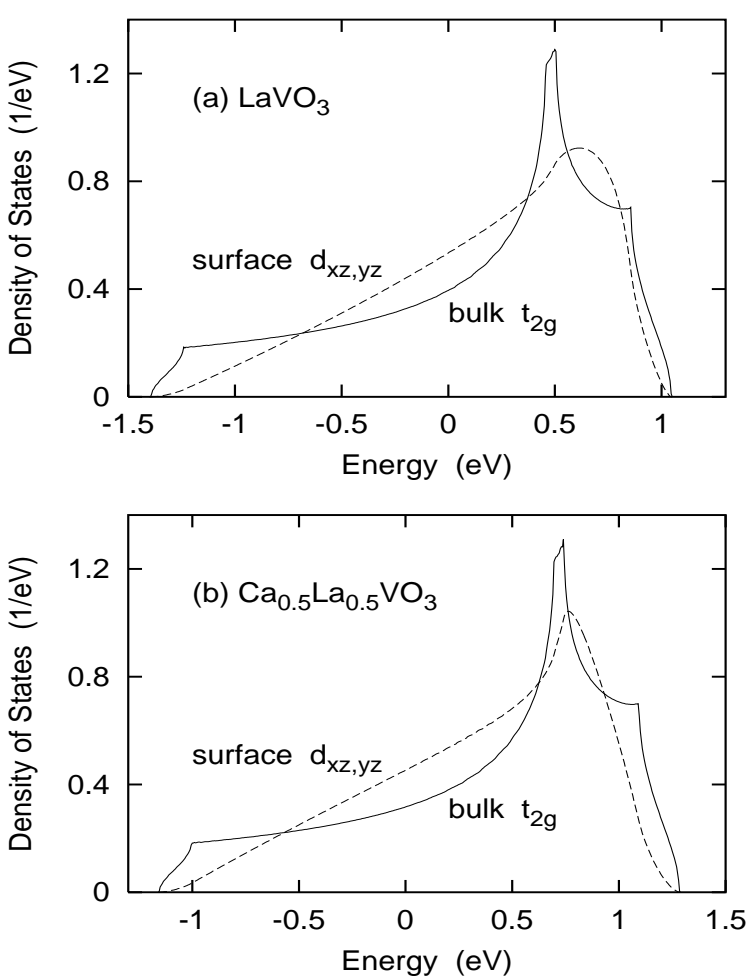

FIG. 7: Solid curves: isotropic bulk density of states of cubic (a) $\mathrm{LaVO}_{3}\left(d^{2}\right)$ and (b) $\mathrm{Ca}_{0.5} \mathrm{La}_{0.5} \mathrm{VO}_{3}\left(d^{1.5}\right)$. Dashed curves: local density of $d_{x z, y z}$ states in the first layer $\left(E_{F}=0\right)$.

tight-binding calculation to ensure charge neutrality at the surface. Qualitatively, however, the densities of both systems exhibit a similar shift of spectral weight from low and high frequencies to the intermediate energy range close to $E_{F}$. Thus, as a result of this effective narrowing of the local density of $d_{x z, y z}$ states we can expect a similar enhancement of surface correlation effects as discussed above for $\mathrm{SrVO}_{3}$ and $\mathrm{SrRuO}_{3}$.

Fig. 8 shows quasi-particle spectra for the bulk and surface layer of $\mathrm{LaVO}_{3}$ at Coulomb energies close to the metal-insulator transition. Since our QMC calculations are carried out at about $1450 \mathrm{~K}(\beta=8)$, the resulting coherent peak near $E_{F}$ obscures the gap between upper and lower Hubbard bands which would appear at low temperatures. Nevertheless, in view of the temperature dependence known from one-band systems [2, 34] the results in Fig. 8 (a) and (b) suggest a bulk Mott transition in the range $U=4.5 \ldots 5.0 \mathrm{eV}$. The surface spectra in Fig. 8 (c) and (d) demonstrate that correlation effects in the first layer are more pronounced than in the bulk, indicating that a gap might form at a slightly lower value of $U$. However, according to the photoemission experiments [5] $\mathrm{LaVO}_{3}$ is insulating in the bulk and at the surface. Thus, we may conclude that within our model $U$ should be about $5 \mathrm{eV}$.

As mentioned above, doping with $\mathrm{Ca}$ makes $\mathrm{LaVO}_{3}$ metallic, leading to rather different excitation spec- 

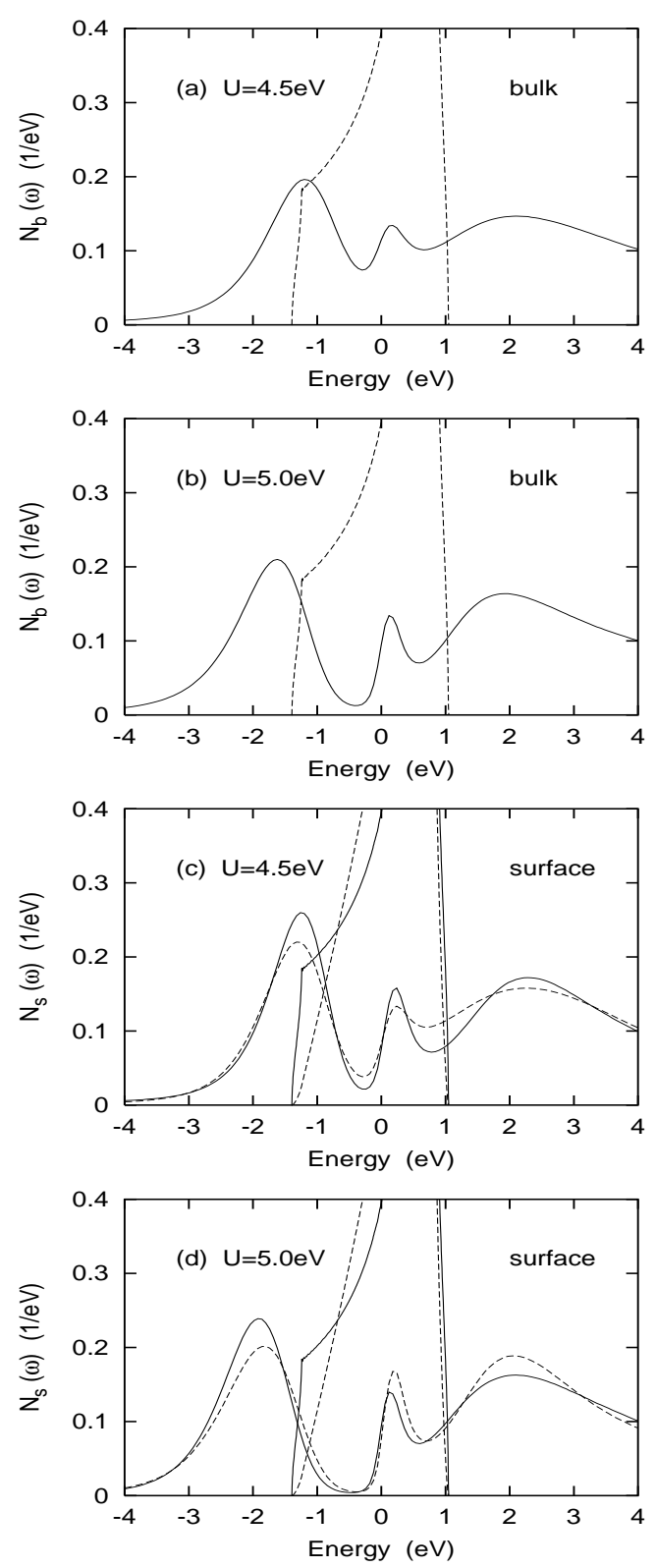

FIG. 8: Quasi-particle density of states $N_{i}(\omega)$ of $\mathrm{LaVO}_{3}$ $\left(d^{2}\right)$ derived from DMFT. (a) and (b): bulk $t_{2 g}$ states for $U=4.5 \mathrm{eV}$ and $U=5.0 \mathrm{eV}$ (solid curves; dashed curves: bare densities). (c) and (d): surface $d_{x y}$ states (solid curves) and $d_{x z, y z}$ states (dashed curves) for $U=4.5 \mathrm{eV}$ and $U=5.0 \mathrm{eV}$; $J=0.7 \mathrm{eV}$.

tra. This drastic change in electronic properties can be seen clearly in the different shapes of the imaginarytime Green's function for $\mathrm{LaVO}_{3}$ and $\mathrm{Ca}_{0.5} \mathrm{La}_{0.5} \mathrm{VO}_{3}$, as shown in Fig. 9. (The value of $G(\tau)$ at $\tau \approx \beta / 2$ is representative of the weight of the quasi-particle peak at $E_{F}$, while $G(\beta)$ gives the occupancy and $G(0)+G(\beta)=-1$ [2].) In the former case, already for $U=4.5 \ldots 5.0 \mathrm{eV}$ there is little weight near $\tau \approx \beta / 2$, indicating the reduction of intensity near $E_{F}$. For $x=0.5$, however, even
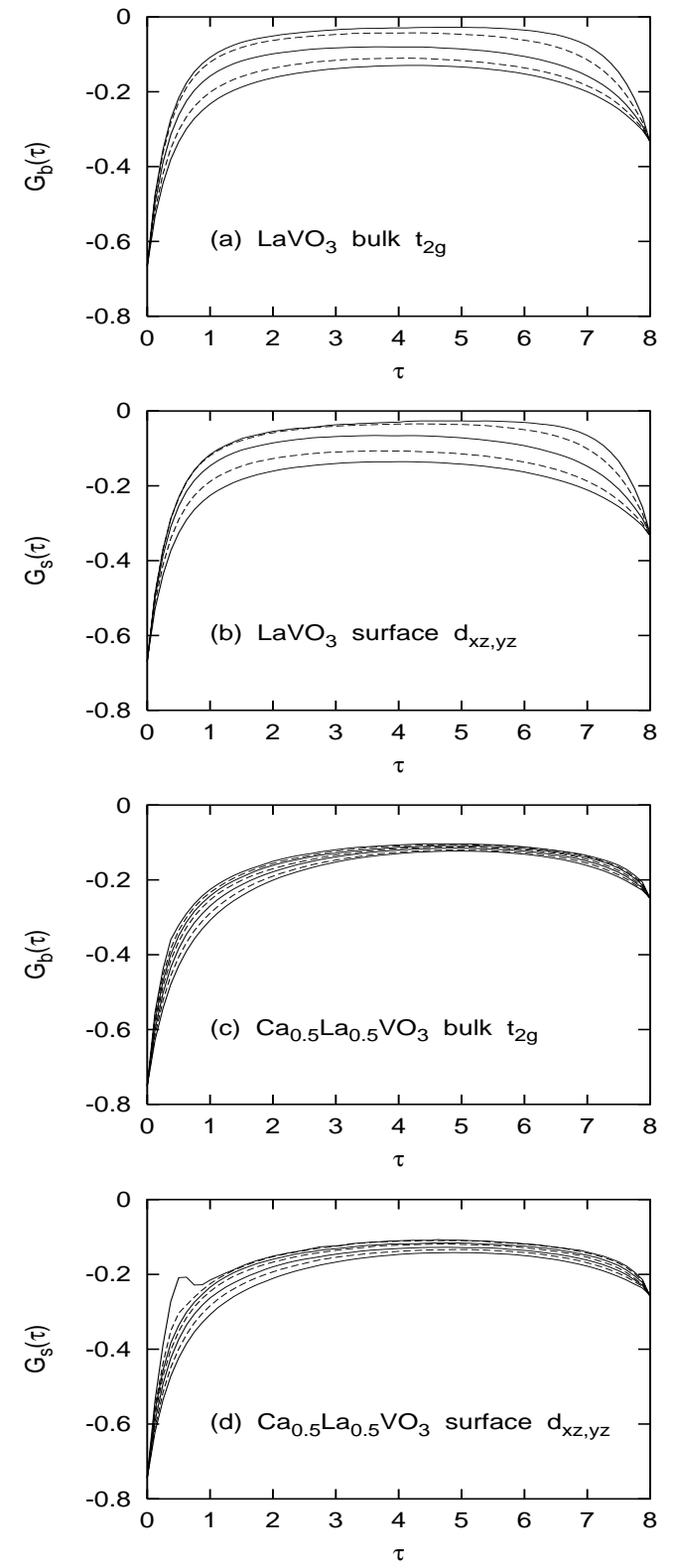

FIG. 9: Quasi-particle Green's function as function of imaginary time derived from DMFT $(\beta=8)$. (a) bulk $t_{2 g}$ states; (b) surface $d_{x z, y z}$ states of $\mathrm{LaVO}_{3}\left(d^{2}\right)$ for $U=3.0 \ldots 5.0 \mathrm{eV}$ in steps of $0.5 \mathrm{eV}$ (from below). (c) bulk $t_{2 g}$ states; (d) surface $d_{x z, y z}$ states of $\mathrm{Ca}_{0.5} \mathrm{La}_{0.5} \mathrm{VO}_{3}\left(d^{1.5}\right)$ for $U=3.0 \ldots 6.0 \mathrm{eV}$ in steps of $0.5 \mathrm{eV}$ (from below). $(J=0.7 \mathrm{eV})$. Note that $\left|G_{i}(\beta / 2)\right|$ is approximately proportional to $N_{i}\left(E_{F}\right)$.

increasing $U$ to $6.0 \mathrm{eV}$ hardly decreases $G(\beta / 2)$. Thus, in the range of Coulomb energies where $\mathrm{LaVO}_{3}$ is clearly insulating, i.e., $U \approx 5.0 \mathrm{eV}, \mathrm{Ca}_{0.5} \mathrm{La}_{0.5} \mathrm{VO}_{3}$ shows robust metallic behavior in the bulk and at the surface, the surface spectra being slightly more correlated than in the bulk. Only for $U=6 \mathrm{eV}$ the surface Green's function for $\mathrm{Ca}_{0.5} \mathrm{La}_{0.5} \mathrm{VO}_{3}$ begins to show a new feature indicating an instability caused by very strong local correlations. 
The calculations discussed above suggest that $\mathrm{LaVO}_{3}$ is an insulator while $\mathrm{Ca}_{0.5} \mathrm{La}_{0.5} \mathrm{VO}_{3}$ is a metal, in agreement experiment. To address the interesting question of separate Mott transitions in the bulk and at the surface, i.e., the possible coexistence of metallic bulk and insulating surface properties, it is necessary to examine the intermediate range of $\mathrm{Ca}$ doping concentrations, $0.0<x<0.5$, and to locate the critical region where the bulk has just become metallic while the surface is still insulating. This issue will be addressed in a subsequent publication.

\section{CONCLUSION}

In summary, we have performed multiband QMCDMFT quasi-particle calculations for several perovskite materials using realistic local densities of states in the bulk and at the surface. As a result of the planar nature of the $t_{2 g}$ states, there is an appreciable effective narrowing of the local density of states in the first layer, even though its total width coincides with the one in the bulk. This band narrowing is entirely a consequence of the reduced atomic coordination at the surface and does not depend on the existence of extra surface states. Typically such states are due to split-off states below or above the main conduction band and would not necessarily contribute to the band narrowing. Other effects, however, which we have not included here, such as tilting or distortion of oxygen octahedra in the surface layer, might well lead to a further reduction of in-plane and/or out-of plane $d-d$ hopping parameters and to additional band narrowing. We also note that the calculations discussed in the present work assume the same value for the Coulomb and exchange energies at the surface as in the bulk. In fact, reduced screening processes close to the surface could cause an increase of $U$ and thereby make surface correlations even more significant. Moreover, the termination of the perovskite surfaces, in particular, the charge state of the transition metal ions, should be investigated.

The main point of our work is that the effective nar- rowing of the surface local density of states leads to an enhancement of correlation effects compared to those in the bulk, in agreement with photoemission data on various systems. Since photoemission spectra always involve bulk as well as surface contributions, it is clearly important to identify the latter in order not to associate them erroneously with enhanced bulk correlations. The pronounced two-dimensional characteristics of $d$ states near $E_{F}$ is a common feature of many transition metal oxides. Thus, the surface-induced enhancement of correlation effects discussed in the present work should be a phenomenon observable in many materials.

We finally remark that all of the systems discussed in the present work have very similar, albeit highly asymmetric bulk densities of states. They differ primarily by the degree of band filling, i.e., by the position of the Fermi level with respect to the density of states features. Also, the surface densities of the out-of-plane subbands of the various systems are qualitatively similar. Nevertheless, even though the quasi-particle spectra for sufficiently large on-site Coulomb energies all exhibit the familiar three-peak structure, with a quasi-particle peak at $E_{F}$ and lower and upper Hubbard satellites, the positions, widths, asymmetric shapes and relative weights of these three peaks vary considerably for the different materials. To describe these variations which evidently reflect the original densities and band fillings, one would need at least 11 parameters (the total area integrates to unity), suggesting that even in the strong-correlation limit the spectra reveal a high degree of system-specific information concerning their electronic structure. Obviously, far greater complexity can be expected for noncubic and magnetic materials. The adequate description of this complexity which lies at the root of many fascinating material properties underlines the tremendous progress that has been achieved by combining density functional theory and quantum impurity methods via the Dynamical Mean Field Theory.

Acknowledgments:

I like to thank A. Bringer for useful discussions and A.I. Lichtenstein for the QMC-DMFT code.
[1] M. Imada, A. Fujimori, and Y. Tokura, Rev. Mod. Phys. 70, 1039 (1998).

[2] A. Georges, G. Kotliar, W. Krauth, and M.J. Rozenberg, Rev. Mod. Phys. 68, 13 (1996).

[3] D. Kalkstein and P. Soven, Surf. Science 26, 85 (1971).

[4] K. Fujioka et al., Phys. Rev. Lett. 56, 6380 (1997).

[5] K. Maiti and D.D. Sarma, Phys. Rev. B 61, 2525 (2000); K. Maiti, P. Mahadevan, and D.D. Sarma, Phys. Rev. Lett. 80, 2885 (1998).

[6] K. Maiti, D.D. Sarma, M.J. Rozenberg, I.H. Inoue, H. Makino, O. Goto, M. Pedio, and R. Cimino, Europhys. Lett. 55, 246 (2001).

[7] A. Sekiyama, H. Fujiwara, S. Imada, H. Eisaki, S.I.
Uchida, K. Takegahara, H. Harima, Y. Saitoh, and S. Suga, cond-mat/0206471

[8] Y. Aiura et al., Phys. Rev. B 47, 6732 (1993).

[9] I.H. Inoue et al., Phys. Rev. Lett. 74, 2539 (1995); Phys. Rev. B 58, 4372 (1998).

[10] K. Morikawa et al., Phys. Rev. B 52, 13711 (1995).

[11] M. Onada, H. Ohta, and H. Nagasawa, Solid State Commun. 79, 281 (1991); M. Kasuya et al., Phys. Rev. B 47, 6193 (1993); A. Fukushima et al., J. Phys. Soc. Jpn. 63, 409 (1994).

[12] I. Nekrasov, G. Keller, D.E. Kondakov, A.V. Kozhevnikov, Th. Pruschke, K. Held, D. Vollhardt, and V.I. Anisimov, cond-mat/0211508 
[13] T. Yokoya et al., Phys. Rev. Lett. 78, 2271 (1977); A.P. Mackenzie et al., Phys. Rev. Lett. 78, 2272 (1997), and references herein.

[14] K.M. Shen et al., Phys. Rev. B 64, 180502(R) (2001); A. Liebsch, Phys. Rev. Lett. 87, 239701 (2001); R. Matzdorf et al., Phys. Rev. B 65, 085404 (2002).

[15] D. Vollhardt, in Correlated Electron Systems, edited by V.J. Emery (World Scientific, Singapore, 1993), p. 57.

[16] Th. Pruschke, M. Jarrell, and J.K. Freericks, Adv. in Phys. 44, 187 (1995).

[17] M. Potthoff and W. Nolting, Phys. Rev. B 60, 7834 (1999), Z. Phys. B 104, 265 (1997).

[18] A. Liebsch, submitted to Phys. Rev. Lett.

[19] K. Takegahara, J. Electron Spectrosc. Relat. Phenom. 66, 303 (1994).

[20] I.H. Inoue, C. Bergemann, I. Hase, and S.R. Julian, Phys. Rev. Lett. 88, 236403-1 (2002).

[21] I.I. Mazin and D.J. Singh, Phys. Rev. B 56, 2556 (1997).

[22] A. Liebsch and A. Lichtenstein, Phys. Rev. Lett. 84, 1591 (2001).

[23] M. Jarrell and J.E. Gubernatis, Phys. Rep. 269, 133
(1996).

[24] J. Kanamori, Progr. Theor. Phys. 30, 275 (1963).

[25] J. Zaanen and G.A. Sawatzky, J. Solid State Chem. 88, 8 (1990); T. Mizokawa and A. Fujimori, Phys. Rev. B 54, 5368 (1996).

[26] E. Müller-Hartmann, Z. Phys. 74, 507 (1989).

[27] I.A. Nekrasov et al., Euro. Phys. J. B 18, 55 (2000).

[28] Y. Maeno, T.M. Rice and M Sigrist, Phys. Today 54, 42 (2001).

[29] M.S. Laad and E. Müller-Hartmann, Phys. Rev. Lett. 87, 246402 (2001).

[30] P. Kostic et al., Phys. Rev. Lett. 81, 2498 (1998).

[31] L. Capogna et al., Phys. Rev. Lett. 88, 076602 (2002).

[32] I. Solovyev, N. Hamada, and K. Terakura, Phys. Rev. B 53, 7158 (1996).

[33] A.E. Bocquet, T. Mizokawa, K. Morikawa, A. Fujimori, S.R. Barman, K. Maiti, D.D. Sarma, Y Tokura, and M. Onada, Phys. Rev. B 53, 1161 (1996).

[34] R. Bulla, T.A. Costi, and D. Vollhardt, Phys. Rev. B 64, 045103 (2001). 